九州大学学術情報リポジトリ

Kyushu University Institutional Repository

\title{
UNIFORM CONVERGENCE OF AN ESTIMATOR OF A DISTRIBUTION FUNCTION
}

Yamato, Hajime

Department of Mathematics, Faculty of Science, Kagoshima University

https://doi.org/10.5109/13073

出版情報: 統計数理研究. 15 (3/4), pp. 69-78，1973-03. Research Association of Statistical Sciences

バージョン :

権利関係 : 


\title{
UNIFORM CONVERGENCE OF AN ESTIMATOR OF A DISTRIBUTION FUNCTION
}

By

\author{
Hajime Yamato*
}

(Received Septemter 27, 1972)

\section{Summary.}

A class of estimators of a distribution function, which includes the empirical distribution function, is discussed. The necessary and sufficient condition for the estimator to be asymptotically unbiased at all continuity points of the distribution function is presented. We give the asymptotic evaluation of the variance and show its asymptotic normality. The necessary and sufficient condition for the estimator to converge uniformly to a continuous distribution function with probability one is presented (the continuity can be delected in case of the necessary condition). We propose an estimator of a $p$-th quantile based on the estimator of a distribution function, which converges to the $p$-th quantile with probability one under certain conditions.

\section{Introduction.}

Let $X_{1}, X_{2}, \cdots, X_{n}$ be a random sample of size $n$ from a population with an unknown distribution function $F(x)$. The empirical distribution function $F_{n}^{*}(x)$ can be expressed as follows,

$$
F_{n}^{*}(x)=\frac{1}{n} \sum_{j=1}^{n} e_{0}\left(x-X_{j}\right),
$$

where $e_{0}$ is the unit distribution function, i. e.,

$$
e_{0}(x)=\left\{\begin{array}{lll}
0 & \text { if } & x<0 \\
1 & \text { if } & x \geqq 0
\end{array}\right.
$$

For any $x, F_{n}^{*}(x)$ is the uniformly minimum variance unbiased estimator of $F(x)$ for all continuous distribution functions, $F$. It is also well known as Glivenko-Cantelli Theorem that

$$
P\left[\sup _{-\infty<x<\infty}\left|F_{n}^{*}(x)-F(x)\right| \rightarrow 0\right]=1 .
$$

Now we shall consider an estimator of the distribution function $F(x)$ given by

$$
F_{n}(x)=-\frac{1}{n} \sum_{j=1}^{n} W_{n}\left(x-X_{j}\right)
$$

\footnotetext{
* Department of Mathematics, Faculty of Science, Kagoshima University, Kagoshima.
} 
where $W_{n}$ is a given distribution function. Obviously the estimator is also a distribution function. In case $F(x)$ is continuous, it seems reasonable to take a continuous distribution function as $W_{n}$ so that we have an continuous estimator $F_{n}(x)$. Particularly in case the distribution function $F(x)$ is absolutely continuous, by taking an absolutely continuous distribution function as $W_{n}, F_{n}(x)$ is again absolutely continuous, which provides an estimator of the density function $f(x)$ as follows,

$$
f_{n}(x)=\frac{1}{n} \sum_{j=1}^{n} w_{n}\left(x-X_{j}\right)
$$

where $w_{n}$ is the derivative of $W_{n}$. After the density estimator of the above form was introduced by Rosenblatt [6], many authors have discussed its statistical properties, for example, Parzen [4], Leadbetter [1], Nadaraya [3], Ryzin [7] and Schuster [9].

For the estimator of a distribution function of the form (1.1), the present author is aware of the following literatures only: Leadbetter [1], Watson and Leadbetter [11] and Yamato [12]. Murthy [2] considered the estimator of the similar form to estimate $1-F(x)$, in which the distribution is absolutely continuous and $w_{n}$ is especially taken as $B_{n} K\left(B_{n}\right)$ with certain restrictions on a constant $B_{n}$ and a density $K$. Leadbetter [1] and Watson and Leadbetter [11], mentioned above, gave the same results for the asymptotic bias, variance and normality in case the distribution is absolutely continuous and $w_{n}$ satisfies certain conditions. In their case $W_{n}(x)$ needs not be a distribution function. It may be natural, however, that $W_{n}$ is a distribution function because it seems favourable if the estimator of a distribution function is also a distribution function.

We shall prepare ourselves with the terminology and notation corcerning the convergence of a sequence of distribution functions. Let $\left\{G_{n}\right\}$ be a sequence of distribution functions and $G$ be a distribution function. Then we say that the sequence $\left\{G_{n}\right\}$ converges completely to $G$ and write

$$
G_{n} \rightarrow G,
$$

if $G_{n}(x) \rightarrow G(x)$ as $n \rightarrow \infty$ at all $x \in C(G)$, where $C(G)$ denotes the set of all continuity points of $G$.

In section 2 , for arbitrarily distribution function it is shown that $F_{n}(x)$ is asymptotically unbiased at all continuity points of the distribution function if and only if $W_{n} \rightarrow e_{0}$. Under the condition $W_{n} \rightarrow e_{0}$ we show that

$$
\lim _{n \rightarrow \infty} n \operatorname{Var}\left[F_{n}(x)\right]=F(x)[1-F(x)] \quad \text { at all } x \in C(F)
$$

and also that the distribution of

$$
\frac{\sqrt{n}\left[F_{n}(x)-E F_{n}(x)\right]}{\sqrt{F(x)[1-F(x)]}}
$$

converges to the normal distribution $N(0,1)$, at all $x \in C(F)$ with $F(x) \neq 0$ or 1 .

In section 3 , we show that if $W_{n} \rightarrow e_{0}$ then the estimator of the form (1.1) converges uniformly to a true continuous distribution function with probability one. It is shown, also, that if the estimator of the form (1.1) converges uniformly to a 
distribution function then we have $W_{n} \rightarrow e_{0}$.

In section 4 , we propose the inverse function of $F_{n}(x)$ as a estimator of a $p$-th quantile, which converges to a unique $p$-th quantile of a continuous distribution function with probability one if $W_{n} \rightarrow e_{0}$. Another applications of the estimator $F_{n}(x)$ will be presented in Yamato [13].

\section{Asymptotic unbiasedness, variance and asymptotic normality.}

LEMMA 1. For any distribution function $F$, the estimator $F_{n}(x)$ of the form (1.1) is asymptotically unbiased at all $x \in C(F)$ if and only if $W_{n} \rightarrow e_{0}$.

Proof. Suppose that $W_{n} \rightarrow e_{0}$. Then the sequence of corresponding characteristic functions $\phi_{W n}(u)$ converges to the unit as $n \rightarrow \infty$ at all points $u$. The expectation

$$
E F_{n}(x)=\int_{-\infty}^{\infty} W_{n}(x-y) d F(y)
$$

is also a distribution function, which is the convolution of the distribution functions $W_{n}$ and $F$. Its characteristic function is denoted by $\phi_{W_{n}}(u) \phi_{F}(u)$, which converges to $\phi_{F}(u)$ as $n \rightarrow \infty$ at all points $u$, where $\phi_{F}$ is the characteristic function of the distribution function $F$. Hence we have

$$
\lim _{n \rightarrow \infty} E F_{n}(x)=F(x) \quad \text { for all } \quad x \in C(F) .
$$

Conversely we suppose that $\lim _{n \rightarrow \infty} E F_{n}(x)=F(x)$ for all $x \in C(F)$, i. e.,

$$
\lim _{n \rightarrow \infty} \int_{-\infty}^{\infty} W_{n}(x-y) d F(y)=F(x) \quad \text { for all } \quad x \in C(F) .
$$

In terms of the characteristic functions the above convergence is equivalent to

$$
\lim _{n \rightarrow \infty} \phi_{W_{n}}(u) \phi_{F}(u)=\phi_{F}(u) \quad \text { for all } u \text {. }
$$

Since $\phi_{F}$ is the characteristic function, $\phi_{F}(u)$ is continuous and equal to unit at $u=0$. Hence there exists a positive constant $\delta$ such that

$$
\phi_{F}(u) \neq 0 \quad \text { on }(-\delta, \delta) .
$$

Therefore by (2.1) and (2.2) we have

$$
\lim _{n \rightarrow \infty} \phi_{W_{n}}(u)=1 \quad \text { for } \quad u \in(-\delta, \delta) .
$$

On the other hand, for any $u$ there exists a positive integer $N$ such that $-\delta<u / N<\delta$ and also we have

$$
\left|\phi_{W_{n}}(u)-1\right|<N\left\{2\left|\phi_{W_{n}}\left(\frac{u}{N}\right)-1\right|\right\}^{1 / 2} .
$$

Since the right hand side of (2.4) converges to zero by (2.3), we have

$$
\lim _{n \rightarrow \infty} \phi_{W n}(u)=1 \quad \text { for all } u,
$$

which implies that $W_{n} \rightarrow e_{0}$ and thus the lemma is proved. 
It is obvious that if $W_{n} \rightarrow e_{0}$ then $W_{n}^{3} \rightarrow e_{0}$. Therefore by the same method as in the proof of the sufficiency of Lemma 1 if $W_{n} \rightarrow e_{0}$ then we have

$$
\lim _{n \rightarrow \infty} \int_{-\infty}^{\infty} W_{n}^{2}(x-y) d F(y)=F(x) \quad \text { for } \quad x \in C(F) .
$$

By Lemma 1 and (2.6), if $W_{n} \rightarrow e_{0}$ then we have

$$
\lim _{n \rightarrow \infty} n \operatorname{Var}\left[F_{n}(x)\right]=F(x)[1-F(x)] \quad \text { for } \quad x \in C(F) .
$$

Consequently if $W_{n} \rightarrow e_{0}$ then we have $\lim _{n \rightarrow \infty} E\left|F_{n}(x)-F(x)\right|^{2}=0$ for $x \in C(F)$ and the estimator $F_{n}(x)$ is consistent for $x \in C(F)$.

THEOREM 1. Let $X_{1}, X_{2}, \cdots, X_{n}$ be independent identically distributed random variables with a distribution function $F(x)$. Suppose that $W_{n} \rightarrow e_{0}$. Then for the estimator $F_{n}(x)$ of the form (1.1) with the above $W_{n}$, the distribution of

$$
\frac{\sqrt{n}\left[F_{n}(x)-E F_{n}(x)\right]}{\sqrt{F(x)[1-F(x)]}}
$$

converges to the normal distribution $N(0,1)$ for all $x \in C(F)$ with $F(x) \neq 0$ or 1 .

Proof. By putting $V_{n j}=W_{n}\left(x-X_{j}\right)$ for $j=1,2, \cdots, n$, our estimator can be expressed as $F_{n}(x)=\frac{1}{n} \sum_{j=1}^{n} V_{n j}$, where $V_{n 1}, \cdots, V_{n n}$ are independent and identically distributed as $V_{n}=W_{n}\left(x-X_{n}\right)$. From (2.7) we have $\lim _{n \rightarrow \infty} \operatorname{Var}\left[V_{n}\right]=\lim _{n \rightarrow \infty} n \operatorname{Var}\left[F_{n}(x)\right]$ $=F(x)[1-F(x)]$ for $x \in C(F)$. On the other hand, by $W_{n}^{3} \rightarrow e_{0}$, we have $\lim _{n \rightarrow \infty} E\left|V_{n}\right|^{3}$ $=\lim _{n \rightarrow \infty} \int_{-\infty}^{\infty} W_{n}^{3}(x-y) d F(y)=F(x)$ for $x \in C(F)$. Henceforth for $x \in C(F)$ we have

$$
\lim _{n \rightarrow \infty} \frac{\sum_{j=1}^{n} E\left|V_{n j}\right|^{3}}{\left\{\sum_{j=1}^{n} \operatorname{Var}\left[V_{n j}\right]\right\}^{3 / 2}}=\lim _{n \rightarrow \infty} \frac{E\left|V_{n}\right|^{3}}{n^{1 / 2}\left\{\operatorname{Var}\left[V_{n}\right]\right\}^{3 / 2^{-}}}=0 .
$$

By Lyapunov's condition the distribution of

$$
\frac{F_{n}(x)-E F_{n}(x)}{\sqrt{\operatorname{Var}\left[F_{n}(x)\right]}}=\frac{\sum_{j=1}^{n}\left[V_{n j}-E V_{n j}\right]}{\sqrt{\sum_{j=1}^{n} \operatorname{Var}\left[V_{n j}\right]}}
$$

converges to the normal distribution $N(0,1)$ for $x \in C(F)$. The above convergence and (2.7) yield the conclusion.

\section{Uniform convergence.}

In case the distribution is absolutely continuous, if the density estimator $f_{n}(x)$ is a density and converges uniformly to the density function with probability one then the corresponding estimator $F_{n}(x)$ of the distribution function converges uniformly to the distribution function with probability one. This follows from the 
fact that if the sequence of density functions $g_{n}(x)$ converges to a density function $g(x)$ almost everywhere then the sequence of corresponding distribution functions converges uniformly to the corresponding distribution function (See, for example, Scheffé [8]). Nadaraya [3] gave a sufficient condition for the density estimator $f_{n}(x)$ of the form (1.2) to converge uniformly to a density function with probability one and Ryzin [7] gave a sufficient condition in multivariate case. It seems to the author that the above two sufficient conditions are different even in univariate case. Schuster [9] presented a necessary and sufficient condition. The sufficient condition given by Nadaraya [3] is the same as the necessary and sufficient condition due to Schuster [9]. In this section we present a sufficient condition and a necessary condition for the estimator $F_{n}(x)$ of the form (1.1) converges uniformly to the distribution function $F(x)$ with probability one.

Let $U$ be the class of all bounded and uniformly continuous real valued functions. Then for a function in $U$ we have the following

LEMMA 2. If $W_{n} \rightarrow e_{0}$, then for any $g \in U$ we have $\lim _{n \rightarrow \infty} \int_{-\infty}^{\infty} g(x) d F_{n}(x)=\int_{-\infty}^{\infty} g(x) d F(x)$ with probability one.

Proof. Since the function $g$ is uniformly continuous and bounded, for any $\varepsilon>0$ there exists a $\delta=\delta(\varepsilon)>0$ such that

$$
|x|<\delta \text { implies }|g(x+y)-g(y)|<\varepsilon \quad \text { for all } y .
$$

There also exists a constant $M>0$ such that

$$
|g(x)| \leqq M \quad \text { for all } x .
$$

As $W_{n} \rightarrow e_{0}$, for the above $\varepsilon>0$ there exists a positive integer $N_{0}=N_{0}(\varepsilon, \delta)$ such that

$$
W_{n}(-\delta)+1-W_{n}(\delta)<\varepsilon \quad \text { for } n>N_{0} .
$$

On the other hand we have

$$
\begin{aligned}
&\left|\int_{-\infty}^{\infty} g(x) d F_{n}(x)-\frac{1}{n} \sum_{j=1}^{n} g\left(X_{j}\right)\right| \\
&=\left|\frac{1}{n} \sum_{j=1}^{n} \int_{-\infty}^{\infty} g\left(x+X_{j}\right) d W_{n}(x)-\frac{1}{n} \sum_{j=1}^{n} g\left(X_{j}\right) \int_{-\infty}^{\infty} d W_{n}(x)\right| \\
& \leqq \frac{1}{n} \sum_{j=1}^{n} \int_{|x| \geq \delta}\left|g\left(x+X_{j}\right)\right| d W_{n}(x)+\frac{1}{n} \sum_{j=1}^{n}\left|g\left(X_{j}\right)\right| \int_{|x| \geqq \delta} d W_{n}(x) \\
&+\frac{1}{n} \sum_{j=1}^{n} \int_{|x|<\delta}\left|g\left(x+X_{j}\right)-g\left(X_{j}\right)\right| d W_{n}(x) .
\end{aligned}
$$

The first and second terms of the last expression of (3.4) are smaller than $M \varepsilon$ for $n>N_{0}$ respectively because of (3.2) and (3.3). The third term is smaller than $\varepsilon$ because of (3.1). Thus (3.4) is smaller than $(2 M+1) \varepsilon$ for $n>N_{0}$. Consequently we have 


$$
\lim _{n \rightarrow \infty}\left\{\int_{-\infty}^{\infty} g(x) d F_{n}(x)-\frac{1}{n} \sum_{j=1}^{n} g\left(X_{j}\right)\right\}=0 .
$$

Since $g$ is bounded, by the strong law of large number we have

$$
\lim _{n \rightarrow \infty} \frac{1}{n} \sum_{j=1}^{n} g\left(X_{j}\right)=\int_{-\infty}^{\infty} g(x) d F(x) \text { with probability one. }
$$

Hence we have

$$
\lim _{n \rightarrow \infty} \int_{-\infty}^{\infty} g(x) d F_{n}(x)=\int_{-\infty}^{\infty} g(x) d F(x) \text { with probability one }
$$

and thus the lemma is proved.

From the above lemma we have the following

LEMMA 3. If $W_{n} \rightarrow e_{0}$, then for all $x \in C(F)$ we have

$$
\lim _{n \rightarrow \infty} F_{n}(x)=F(x) \text { with probability one. }
$$

Proof. Let $\left\{a_{m}\right\}$ be a sequence of positive numbers, which converges to zero. Let the function $g_{1 m}(t)$ for all positive integer $m$ be such that

$$
g_{1 m}(t)= \begin{cases}1 & \text { if } t \leqq x-a_{m} \\ 0 & \text { if } t \geqq x\end{cases}
$$

and $g_{1 m}(t)$ is continuous on $\left[x-a_{m}, x\right]$. Let the function $g_{2 m}(t)$ for all positive integer $m$ be such that

$$
g_{2 m}(t)= \begin{cases}1 & \text { if } t \leqq x \\ 0 & \text { if } t \geqq x+a_{m}\end{cases}
$$

and $g_{2 m}$ is continuous on $\left[x, x+a_{m}\right]$. It is obvious that $g_{1 m}, g_{2 m} \in U$ for any $m$. Hence by Lemma 2, we have for any $m$

$$
\lim _{n \rightarrow \infty} \int_{-\infty}^{\infty} g_{1 m}(t) d F_{n}(t)=\int_{-\infty}^{\infty} g_{1 m}(t) d F(t)
$$

and

$$
\lim _{n \rightarrow \infty} \int_{-\infty}^{\infty} g_{2 m}(t) d F_{n}(t)=\int_{-\infty}^{\infty} g_{2 m}(t) d F(t)
$$

on $N_{x, m}^{c}$, where $N_{x, m}$ is a set with $P\left(N_{x, m}\right)=0$ and $N_{x, m}^{c}$ is the complement of $N_{x, m}$. Now let us put $N_{x}=\bigcup_{m=1}^{\infty} N_{x, m}$. Then $P\left(N_{x}\right)=0$ and we have (3.5) and (3.6) on $N_{x}^{c}$ for any $m$. By the definition of $g_{1 m}$ and $g_{2 m}$, we have

$$
\left\{\begin{array}{l}
F\left(x-a_{m}\right) \leqq \int_{-\infty}^{\infty} g_{1 m}(t) d F(t) \\
\int_{-\infty}^{\infty} g_{1 m}(t) d F_{n}(t) \leqq F_{n}(x) \leqq \int_{-\infty}^{\infty} g_{2 m}(t) d F_{n}(t) \\
\int_{-\infty}^{\infty} g_{2 m}(t) d F(t) \leqq F\left(x+a_{m}\right) .
\end{array}\right.
$$


The combination of (3.5), (3.6) and (3.7) yields for any $m$

$$
F\left(x-a_{m}\right) \leqq \liminf _{n} F_{n}(x) \leqq \limsup _{n} F_{n}(x) \leqq F\left(x+a_{m}\right) \text { on } N_{x}^{c} .
$$

By letting $m$ tend to infinity in the above inequality, if $x \in C(F)$ then we have

$$
\lim _{n \rightarrow \infty} F_{n}(x)=F(x) \quad \text { on } \quad N_{x}^{c} .
$$

Thus the lemma is proved.

THEOREM 3. Let $X_{1}, X_{2}, \cdots, X_{n}$ be independent identically distributed random variables with a distribution function $F(x)$. If $W_{n} \rightarrow e_{0}$ and $F(x)$ is continuous then for the estimator $F_{n}(x)$ given by (1.1) with the above $W_{n}$, we have

$$
P\left[\sup _{-\infty<x<\infty}\left|F_{n}(x)-F(x)\right| \rightarrow 0\right]=1 .
$$

Conversely for any distribution function $F(x)$ if (3.8) holds then we have $W_{n} \rightarrow e_{0}$.

PROOF. If $W_{n} \rightarrow e_{0}$ and $F(x)$ is continuous then by lemma 3 we have $F_{n}(x) \rightarrow F(x)$. with probability one for all $x$. Therefore by giving the same proof as of GlivenkoCantelli Theorem (See, for example, Tucker [10], pp. 127-128), we can show (3.8).

Conversely suppose that (3.8) holds for any distribution function. Then by this assumption and Glivenko-Cantelli Theorem there exists a set $N$ with $P(N)=0$ such that

$$
\begin{cases}\lim _{n \rightarrow \infty} F_{n}(x)=F(x) & \text { for all } x \\ \lim _{n \rightarrow \infty} F_{n}^{*}(x)=F(x) & \text { for all } x\end{cases}
$$

on $N^{c}$. Let $\phi_{F_{n}}$ and $\phi_{F_{n}^{*}}$ denote the characteristic function of distribution functions. $F_{n}$ and $F_{n}^{*}$ respectively. Then we have

$$
\phi_{F_{n}}(u)=\frac{1}{n}-\sum_{j=1}^{n} \int_{-\infty}^{\infty} e^{i u\left(x+X_{j}\right)} d W_{n}(x)=\phi_{F_{n}^{*}}(u) \phi_{W_{n}}(u)
$$

and from (3.9) we have

$$
\begin{cases}\lim _{n \rightarrow \infty} \phi_{F_{n}}(u)=\phi_{F}(u) & \text { for all } u \\ \lim _{n \rightarrow \infty} \phi_{F_{n}^{*}}(u)=\phi_{F}(u) & \text { for all } u\end{cases}
$$

on $N^{c}$. On the other hand since $\phi_{F}$ is a characteristic function, there exists a positive constant $\delta$ such that

$$
\phi_{F}(u) \neq 0 \quad \text { for } \quad u \in(-\delta, \delta) .
$$

From (3.10), (3.11) and (3.12), we have

$$
\lim _{n \rightarrow \infty} \phi_{W_{n}}(u)=1 \quad \text { for } \quad u \in(-\delta, \delta) \text { on } N^{c} .
$$

Since the characteristic function $\phi_{W_{n}}$ does not depend on $N$, we have

$$
\lim _{n \rightarrow \infty} \phi_{W_{n}}(u)=1 \quad \text { for } \quad u \in(-\delta, \delta) .
$$


Therefore by following the same discussion as from (2.3) to (2.5) we have

$$
\lim _{n \rightarrow \infty} \phi_{W_{n}}(u)=1 \quad \text { for all } u \text {. }
$$

which is equivalent to $W_{n} \rightarrow e_{0}$. Thus the theorem is proved.

Alternatively the first part of Theorem 2 can also be proved as follows: if $W_{n} \rightarrow e_{0}$ then we have

$$
\lim _{n \rightarrow \infty} \dot{\phi}_{W_{n}}(u)=1 \quad \text { for all } u
$$

and by Glivenko-Cantelli Theorem we have

$$
\lim _{n \rightarrow \infty} \dot{\phi}_{F_{n}^{*}}(u)=\dot{\phi}_{F}(u) \quad \text { for all } u \text { on } N^{c} \text {, }
$$

where $N$ is a set with $P(N)=0$. Consequently by (3.10) we have

$$
\lim _{n \rightarrow c} \phi_{F n}(u)=\dot{\phi}_{F}(u) \quad \text { for all } u \text { on } N^{c} \text {. }
$$

Therefore we have

$$
\lim _{n \rightarrow \infty} F_{n}(x)=F(x) \quad \text { for all } x \in C(F) \text { on } N^{c} .
$$

If $F(x)$ is continuous, then the above convergence holds uniformly on $N^{c}$ and we have

$$
\left.\limsup _{n \rightarrow \infty} \mid F_{n}(x)-F(x)\right]=0 \quad \text { on } \quad N^{c}
$$

\section{Estimation of a quantile.}

An estimator of a distribution function can be used to estimate a median, a lower quantile, a upper quantile and in general, a $p$-th quantile. The estimator $F_{n}(x)$ of the form (1.1) yields estimators $F_{n}^{-1}(1 / 2), F_{n}^{-1}(1 / 4), F_{n}^{-1}(3 / 4)$ and $F_{n}^{-1}(p)$, which are estimators of a median, a lower quantile, a upper quantile and a $p$-th quantile respectively, where $F_{n}^{-1}$ is the inverse function of $F_{n}$ defined by $F_{n}^{-1}(u)=\sup \{x$ : $\left.F_{n}(x)<u\right\}$ and $0<p<1$. These estimators, in general, can not be expressed in a explicit form, but are easily obtained by drawing the curve $y=F_{n}(x)$ on the $(x, y)$ plane, for example using the computer plotting device, and reading the $x$-axis of the intersection of the above curve and lines, $y=0.5,0.25,0.75$ and $p$. In case there does not exist such intersection, we should read the supremum of the $x$-axis giving the maximum value of $F_{n}(x)$ which does not exceed $y=0.5,0.25,0.75$ and $p$. In case the $x$-axis of such intersection consists of an interval, we should read the infimum of the interval. Especially if we taken $F_{n}(x)$ with $W_{n}=e_{0}$ for all $n$, i. e., the empirical distribution function $F_{n}^{*}(x)$, then we can express $F_{n}^{-1}(p)$ in a familiar form. Let $X_{(1)}, \cdots, X_{(n)}$ be the order statistics of the sample $X_{1}, \cdots, X_{n}$. Then the estimator $F_{n}^{-1}(p)$ with $W_{n}=e_{0}$ for all $n$, i. e., $F_{n}^{*-1}(p)$, is equal to $X_{\langle k\rangle}$ if $p=k / n$ and equal to $X_{(k+1)}$ if $k / n<p<(k+1) / n$, where $k$ is a positive integer smaller than $n$. If there is a unique $p$-th quantile of a distribution, then this estimator converges to the $p$-th quantile with probability one (See, for example, proposition (i) in Rao [5], pp. 355). We show in Proposition 2 that if there exists a unique $p$-th quantile of a continuous 
distribution function and $W_{n} \rightarrow e_{0}$, then the estimator $F_{n}^{-1}(p)$ converges to the $p$-th quantile with probability one. In order to estimate a $p$-th quantile of a continuous distribution function, the estimator $F_{n}^{-1}(p)$ with a suitably chosen continuous distribution function $W_{n}$ will be recommended over the estimator $F_{n}^{*-1}(p)$.

Proposition 1. Suppose $W_{n} \rightarrow e_{0}$. Let $F$ be a continuous distribution function and $0<p<1$. Let $F_{n}^{-1}$ be the inverse function of $F_{n}$, where $F_{n}$ is given by (1.1) with the above $W_{n}$. Then we have

$$
\sup \{\xi: F(\xi)<p\} \leqq \lim _{n} \inf F_{n}^{-1}(p) \leqq \lim _{n} \sup F_{n}^{-1}(p) \leqq \inf \{\xi: F(\xi)>p\}
$$

with probability one.

Proof. By the assumption the distribution function $F(x)$ is strictly increasing on $(a-\varepsilon, a]$ for any $\varepsilon>0$, where $a=\sup \{\xi: F(\xi)<p\}$. Therefore we have for the above $\varepsilon>0$

$$
F\left(a-\frac{\varepsilon}{2}\right)<p
$$

Hence from Theorem 2 for sufficiently large $n$ we have

$$
F_{n}\left(a-\frac{\varepsilon}{2}\right)<p
$$

with probability one. Since $F_{n}$ is a distribution function, for sufficiently large $n$ we have

$$
a-\varepsilon<a-\frac{\varepsilon}{2} \leqq F_{n}^{-1}(p)
$$

with probability one. Therefore we have

$$
\lim _{n} \inf F_{n}^{-1}(p) \geqq a \text { with probability one. }
$$

Similarly we can also show

$$
\lim \sup _{n} F_{n}^{-1}(p) \leqq \inf \{\xi: F(\xi)>p\} \quad \text { with probability one. }
$$

If in the above proposition especially there exists a unique $\xi$ satisfying $F(\xi)=p$, then we have the following

Proposition 2. Suppose that there exists a unique p-th quantile $\xi$. Then under the same conditions as Proposition 1, we have

with probability one.

$$
\lim _{n \rightarrow \infty} F_{n}^{-1}(p)=\xi
$$

The above proposition shows that the estimator $F_{n}(x)$ of a continuous distribution function yields a consistent estimator $F_{n}^{-1}(p)$ of a unique $p$-th quantile.

The author wishes to thank Prof. A. Kudo of Kyushu University for his encouragement and advices. 


\section{References}

[1] LeAdBetter, M. R. (1963), On the non-parametric estimation of probability densities, Technical Report No. 11, Research triangle Institute.

2 Mtrthy, V.K. (1965), Estimation of jumps, reliability and hazard rate, Ann. Math. Statist., Vol. 36, pp. 1032-1040.

[- 3$]$ NADARAYA, E. A. (1965), On non-parametric estimates of density functions and regression curves, Theor. Prob. Appl. Vol. 10, pp. 186-190.

[4] Parzex, E. (1962), On estimation of a probability density function and mode, Ann. Math. Statist., Vol. 33, pp. 1065-1076.

-5] RAO, C. R. (1967), Linear Statistical inference and its applications, John Wiley, New York.

[6] RosenblRTt, M. (1956), Remarks on some non-parametric estimates of a density function, Ann. Math. Statist., Vol. 27, pp. 832-837.

[7] RYZIN, J. VAN (1969), On strong consistency of density estimates, Ann. Math. Statist., Vol. 40, pp. 1765-1772.

[8] Scheffé, H. (1947), A useful convergence theorem for probability distributions, Ann. Math. Statist., Vol. 18, pp. 434-438.

[9] Schlister, E. F. (1970), Note on the uniform convergence of density estimates, Ann. Math. Statist., Vol. 41, pp. 1347-1348.

[10] Tucker, H. G. (1967), A graduate course in probability, Academic Press, New York.

11] Watson, G.S. and Leadbetter, M. R. (1964), Hazard Analysis, II, Sankhyā, Ser. A, Vol. 26, pp. 101-116.

[12] Yamato, H. (1972), Some statistical properties of estimators of density and distribution functions, Bull. Math. Statist., pp. 113-131.

[13] Yamato, H. (1972), Discrimination and estimating the difference in location parameters (to appear in Rep. Fac. Sci. Kagoshima Univ.). 